\title{
Experimental Protocol of Accuracy Evaluation of 6-D Localizers for Computer-Integrated Surgery: Application to Four Optical Localizers
}

\author{
Fabrice Chassat, Stephane Lavallée, Ph.D. \\ TIMC Laboratory, Faculté de Médecine, I.A.B., 38706 La Tronche France \\ e-mail : Fabrice.Chassat@imag.fr
}

\begin{abstract}
This paper describes an experimental protocol in order to evaluate the accuracy of localizers for computer-integrated surgery (CIS), and the result of the evaluation for four commercially available optical localizers (Optotrak, FP5000, Polaris active and passive). This paper shows that the four systems have static xyz accuracy compatible with most of CIS applications, but they significantly differ when comparing their accuracy of moving rigid bodies.
\end{abstract}

\section{Introduction}

In CIS, the six degrees of freedom (6D) localization is used to locate and follow in space the position and orientation of rigid bodies constituted of markers, with respect to a reference rigid body. For example, a localizer can be used to track the tip and the axis of a pointer or surgical tool on pre-operative images (after registration) or to digitize landmarks or surfaces on anatomical structures to track the position of the sensor such as an ultrasound probe, or to measure the relative positions of bones $[1,2,3]$,

An extensive list of commercial products existing for localization can be found in $[4,5]$, but few of them meet the CIS requirements in terms of accuracy (about $1 \mathrm{~mm}$ in $1 \mathrm{~m}^{3}$ ), reliability, clinical usability... Four technologies have been used in CIS :

Mechanical technology : Mechanical localizers such as Viewing-Wands ${ }^{T M}$ use a 6 -axes coding robot-arm $[6,7]$. The main problem of this technology is the global clutter if we need to locate more than one rigid body.

Magnetical technology : Magnetical localizers such as Polhemus ${ }^{T M}$ or Flocks-of-Birds ${ }^{T M}$ use a magnetic source and a receiver of magnetic fields [8]. The problem of this technology is the relative inaccuracy due to metallic objects. At least, those systems imply to use non-ferromagnetic objects in the environment.

Acoustical technology : Acoustical localizers such as Pegasus ${ }^{T M}$ measure the time of flight between emitters and receivers in order to get distance information. Unfortunately, measurements are sensitive to several perturbation such as air movements, parasite reflexions, temperature gradients in space and time [9].

Optical technology : In this category ther are the most usefull localizers 
for surgery because of their good accuracy and good reliability [10]. However, it is important to notice that optical localizers raise the problem of visibility between the markers that constitute the rigid bodies and the cameras. Some systems use three linear CCD cameras and infrared LEDs plugged in rigid bodies. The position measurement is made by computing the intersection of the three planes corresponding to each camera. Other systems use a classical stereovision principle to locate markers which can be active like LEDs, or passive like retroreflective spheres or patterns [11]. Each camera gives a three dimension line of view. The intersection of two 3D lines gives a point. For both technologies, rigid bodies transformations are computed from individual markers locations using paired-points matching algorithms [12].

\section{Experimental protocol for localizer evaluation}

\subsection{Intrinsic Accuracy Test (I.A.T.)}

Here, we characterize the intrinsic accuracy of the localizer that is important to obtain an upper bound of the useful accuracy. One marker is located with respect to the coordinate system associated with the localizer. An experiment corresponding to this test is presented in Fig.1. We use a $1 \mathrm{~m}$ motorized linear displacement system which can perform displacements of $0.1 \mathrm{~mm}$. And we measure the position of 100 points $M_{i}$, where each point is separated from his neighbours $\mathrm{M}_{i-1}$ and $\mathrm{M}_{i+1}$ by a true distance of $10 \mathrm{~mm}$. The $x$ axis is the horizontal displacement (right to left from the cameras at a $1 \mathrm{~m} 90$ depth to the cameras), the $y$ axis is the depth displacement from $1 \mathrm{~m} 90$ to $2 \mathrm{~m} 90$ with respect to cameras, and the $z$ axis is the vertical displacement at a $1 \mathrm{~m} 90$ depth to the cameras.

On each series of 100 points $\mathrm{M}_{i}$ we check that

$e_{i}=\left\|M_{i}-M_{\text {center }}\right\|-i .10 \mathrm{~mm}=0$, where $\mathbf{M}_{\text {center }}$ is the point corresponding to the middle of the linear displacement system. Then, we calculate for $\mathrm{e}_{i}$ the mean error $: \bar{e}=\frac{1}{N} \sum_{i=1}^{N} e_{i}$, the rms error $: r m s=\sqrt{\frac{1}{N} \sum_{i=1}^{N}\left(e_{i}-\bar{e}\right)^{2}}$, the $\max$ error : $\operatorname{Max}\left|e_{i}\right|$.

\subsection{Relative Rigid Body Accuracy Test (R.R.B.A.T.)}

Here we characterize the accuracy of the localizers for the relative measurement between two rigid bodies. They are placed in a common rigid support as represented in Fig.2. With the localizer, we get the transformation matrices $\mathbf{T}_{0} \ldots$ $\mathrm{T}_{N}$ from Ref $\mathrm{f}_{\text {body }}$, which are associated to one rigid body, to $\operatorname{Ref}_{\text {sensor }}$ which is associated to the other rigid body. Considering $\left(\mathrm{x}_{i}, \mathrm{y}_{i}, \mathrm{z}_{i}\right)$ as the coordinates of the origin point of $\operatorname{Ref}_{\text {body }}$ in $\operatorname{Ref}_{\text {sensor }}$ we check the stability of those transformation matrices by computing the distances :

$\mathrm{d}_{i}=\sqrt{\left(x_{i}-\bar{x}\right)^{2}+\left(y_{i}-\bar{y}\right)^{2}+\left(z_{i}-\bar{z}\right)^{2}}$, where $\bar{x}, \bar{y}, \bar{z}=\frac{1}{N} \sum_{i=1}^{N} x_{i}, y_{i}, z_{i}$ between the two systems of reference. Then, stability of the rotation angle $\alpha_{i}$ between

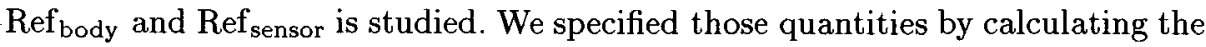
max and rms error on $\mathrm{d}_{i}$ and $\alpha_{i}$. 
This test is performed with 8 different conditions, with $\mathrm{N}=100$ and the support in a plane roughly parallel to the cameras except for test IV :

I static on identical positions.

II static on different positions.

III hand-holding on identical positions.

IV hand-holding on different positions with a random angle of the support with respect to cameras.

$\mathbf{V}$ dynamic in translation at a constant low speed.

VI dynamic hand-holding with circular motions at low speed on, that is to say that the support is making circles very slowly around an axis which is orthogonal to the rigid bodies support.

VII dynamic hand-holding with circular motions at medium speed.

VIII dynamic hand-holding with circular motions at high speed.

\subsection{Pivot Repetability Test (P.R.T.)}

We are interested in evaluating the accuracy of the determination of the tip of a pointer (attached to a rigid body Ref $_{\text {sensor }}$ ) with the pivot method [13]. A sapphire sphere is placed at the tip of the pointer and is put in a small hole. We slowly rotate the pointer around the sphere and get 50 positions of $\operatorname{Ref}_{s}$ ensor, then we find the location of the most invariant point in the coordinate system associated with a rigid body attached to the hole, as presented in Fig.3. The pivot test provides an invariant point with residual errors of the 50 measures: rms $_{\text {pivot }}$ and $\max _{\text {pivot }}$. The xyz coordinates of this invariant point should be independant of the experiment, thus we repeat the pivot measurements for 20 experiments. So we could measure a mean point and the $\mathrm{rms}_{\text {repeat }}$ and max $_{\text {repeat }}$ deviation from this mean point.

\subsection{Surface Digitization Accuracy Test (S.D.A.T.)}

To evaluate accuracy of surface digitization, it is first necessary to calibrate the pointer. It is done using the precedent pivot method with $N=1000$ rotating positions. Then, 100 predefined points of the surface of a plane (which is a $14 \mu \mathrm{m}$ stabilized surface) are collected (see Fig.4). With these points, we fit a least-squares plane and determine the residual distance $d_{i}$ of each point to the least-squares plane. We study the rms and max error in $\mathrm{mm}$ of $\left|d_{i}\right|$.

\section{Evaluation of four optical localizers}

In this section, we present the results of the experimental protocol that is defined in section 2 for four commercially available optical localizers :

Optotrak $^{T M}$ (Northern Digital Inc. Ontario Canada) which is a system using 3 linear cameras. (This system was acquired in 1992 and never revised.) (Fig.5) FP5000 ${ }^{T M}$ (Image Guided Technologies. Boulder, Colorado USA) which is also a system with 3 linear cameras. (1996). (Fig.6) 


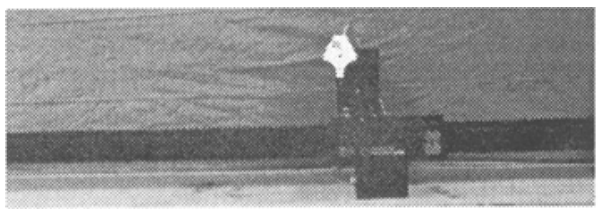

Fig. 1. I.A.T. protocol

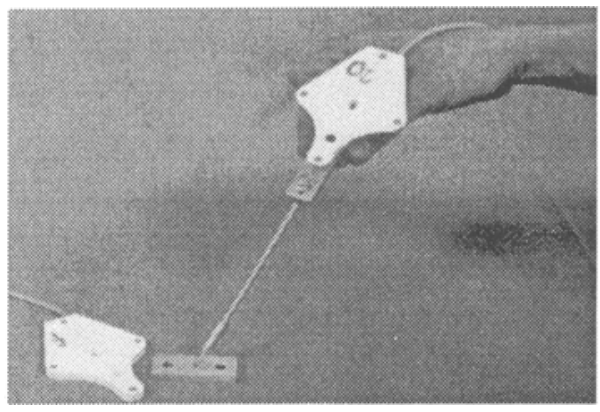

Fig. 3. P.R.T. protocol

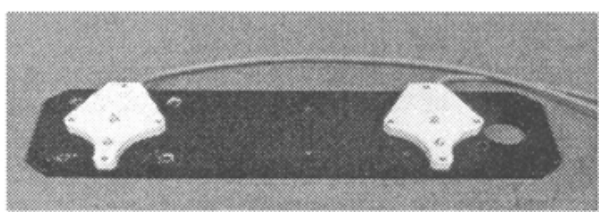

Fig. 2. R.R.B.A.T. protocol

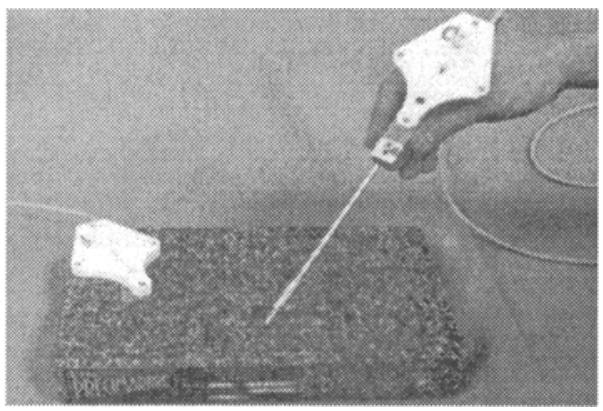

Fig. 4. S.D.A.T. protocol

Active Polaris $^{T M}$ (Northern Digital Inc. Ontario Canada) which is a system using two 2-D cameras. (1997). (Fig.7)

Passive Polaris $^{T M}$ (Northern Digital Inc. Ontario Canada) which is a system using two 2-D cameras. (1997). Passive Polaris is in fact an hybrid system that can track active or passive markers indifferently, but we evaluated only the passive mode of this system. (Fig.7)

All those localizers produce measurements at a frame rate compatible with most of CIS applications. Although, the Optotrak system has high speed measurement capabilities, this was not considered as an important criteria for C.I.S. The size of rigid bodies are roughly similar for the four evaluated localizers.

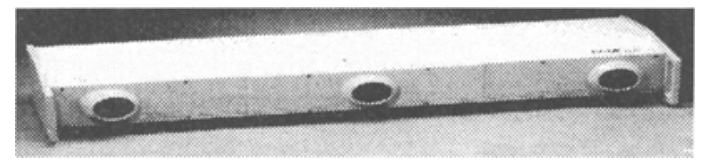

Fig. 5. Optotrak device

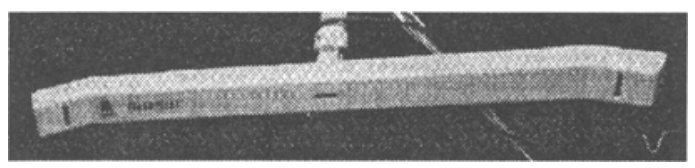

Fig. 6. FP5000 device

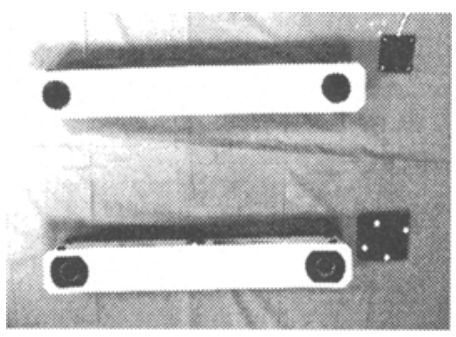

Fig. 7. Polaris devices and rigid bodies (up : Active system, down : Passive system) 
Every values concerning distances are in $\mathrm{mm}$, while every values concerning angles are in degrees. The results of the I.A.T. tests are not numerically mentionned but are discussed in the next section. The results of the R.R.B.A.T. tests are in Table 1, results of the P.R.T. are in Table 2, results of the S.D.A.T. are in Table 3 .

Table 1. Relative Rigid Body Accuracy Test (distances values are in mm, angle values are in degrees)

\begin{tabular}{|c|c|c|c|c|c|}
\hline \multirow{5}{*}{ Static measure } & \multicolumn{5}{|c|}{ 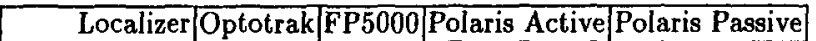 } \\
\hline & $\overline{d_{\max }}$ & $0 . \overline{888}$ & 1.764 & $\overline{1.062}$ & 0.624 \\
\hline & rms error on $d$ & 0.067 & 0.408 & 0.189 & 0.1431 \\
\hline & $\alpha_{\max }$ & 0.020 & 0.337 & 0.355 & 0.1484 \\
\hline & rms error on $\alpha$ & 0.009 & 0.141 & 0.126 & 0.0536 \\
\hline Static measure in & $d_{\max }$ & 1.052 & 2.825 & 3.156 & 0.712 \\
\hline diferents posititons & rms error on d & 0.220 & 0.571 & 0.653 & 0.172 \\
\hline & $\alpha_{\max }$ & 0.071 & 0.649 & 0.863 & 0.150 \\
\hline 11 & rms error on $\alpha$ & 0.026 & 0.275 & 0.279 & 0.055 \\
\hline Static measure in & $d_{\max }$ & 0.400 & 1.424 & 3.253 & 1.026 \\
\hline hand-holding & rms error on $d$ & 0.098 & 0.331 & 0.432 & 0.241 \\
\hline & $\alpha_{\max }$ & 0.035 & 0.422 & 0.578 & 0.168 \\
\hline III & rms error on $\alpha$ & 0.011 & 0.104 & 0.204 & 0.057 \\
\hline Static measure in & $\overline{d_{\max }}$ & $0 . \overline{883}$ & 3.035 & 9.179 & 2.026 \\
\hline diferents posititons & rms error on $d$ & 0.149 & 0.621 & 2.073 & 0.405 \\
\hline in hand-holding & $\alpha_{\max }$ & 0.050 & 0.504 & 0.771 & 0.388 \\
\hline IV & rms error on $\alpha$ & 0.015 & 0.183 & 0.240 & 0.101 \\
\hline Dynamic measure in & $d_{\max }$ & 1.297 & 1.820 & 3.729 & 2.100 \\
\hline translation at constant speed & rms error on d & 0.225 & 0.415 & 0.620 & 0.451 \\
\hline & $\boldsymbol{\alpha}_{\max }$ & 0.096 & 0.501 & 0.796 & 0.216 \\
\hline V & rms error on $\alpha$ & 0.026 & 0.191 & 0.260 & 0.065 \\
\hline Dynamic measure in & $d_{\max }$ & 0.560 & 1.855 & 6.526 & 1.838 \\
\hline rotation at low speed & rms error on d & 0.126 & 0.337 & 0.961 & 0.346 \\
\hline & $\alpha_{\max }$ & 0.059 & 0.687 & 0.585 & 0.166 \\
\hline VI & rms error on $\alpha$ & 0.015 & 0.236 & 0.207 & 0.061 \\
\hline Dynamic measure in & $\overline{d_{\max }}$ & 0.575 & 2.180 & 9.205 & 1.839 \\
\hline rotation at medium speed & rms error on $d$ & 0.114 & 0.453 & 1.161 & 0.362 \\
\hline & $\alpha_{\max }$ & 0.059 & 0.657 & 0.724 & 0.166 \\
\hline VII & rms error on $\alpha$ & 0.021 & 0.160 & 0.202 & 0.065 \\
\hline Dynamic measure in & $d_{\max }$ & 0.521 & 5.100 & 23.881 & 1.622 \\
\hline rotation at high speed & rms error on $d$ & 0.107 & 0.628 & 4.639 & 0.314 \\
\hline & & 0.063 & 1.795 & 0.811 & 0.203 \\
\hline VIII & rms & 0.024 & 0.521 & 0.232 & 0.080 \\
\hline
\end{tabular}


Table 2. Pivot Repeatability Test, every values are in $\mathrm{mm}$

\begin{tabular}{|r|c|c|c|c|}
\hline Localizer & Optotrak & FP5000 & Polaris Active & Polaris Passive \\
\hline \hline Error : $\max \left({\left.\text { error } \text { max }_{\text {pivot }}\right)}\right)$ & 0.683 & 9.101 & 2.201 & 5.217 \\
mean(rms pivot $_{\text {error }}$ & 0.102 & 0.813 & 0.385 & 0.608 \\
\hline Distance : rms $_{\text {repeat }}$ & 0.313 & 0.492 & 0.248 & 0.312 \\
max $_{\text {repeat }}$ & 0.478 & 0.815 & 0.434 & 0.594 \\
\hline
\end{tabular}

Table 3. Surface Digitization Accuracy Test, every values are in $\mathrm{mm}$

\begin{tabular}{|r|c|c|c|c|}
\hline Localizer & Optotrak & FP5000 & Polaris Active & Polaris Passive \\
\hline \hline rms distance & 0.058 & 0.709 & 0.593 & 0.253 \\
\hline max distance & 0.249 & 1.852 & 4.819 & 0.755 \\
\hline
\end{tabular}

\section{Discussion}

Tests I.A.T. (intrinsic accuracy) : It appears that the Optotrak device is the most accurate system for this test, but all localizers have enough intrinsic accuracy for CIS applications. We can notice for each localizer a maximum error for the displacement in direction y (depth).

Actually, this test only provides a lower bound of errors, the values are indeed in the same range that the linear displacement accuracy that we used.

Tests R.R.B.A.T. (relative accuracy) : To conclude on those tests, we can say that the Optotrak and the Passive Polaris have better accuracy than the two others. The FP5000 gives sufficient accuracy for most of the CIS applications but in some particular applications it is unusable. The Active Polaris, due to its data acquisition protocol (it pulses all the LEDs corresponding to one rigid body and at the next step all the LEDs corresponding to the other rigid body) provides very bad results when we get motion positions and is therefore unusable in those cases. Nevertheless, the relative accuracy in static position of the Active Polaris is similar to the FP5000.

Tests P.R.T. (pivot) : The global results on the different positions of the tip of the pointer are all similar and all good. Optotrak is the only device which is able to have a very stable error. The other devices can make ponctual important errors which do not really influence the final result since it is average on a large number of data.

Tests S.D.A.T. (planar surface) : As for the three others tests, the Optotrak gives the best results. In fact, all results for this tests are acceptable even if the Active Polaris has a maximum error which can raise problems in some cases.

General discussion : According to our results, we can say that those four localizers are raisonnably adapted for surgery, but care should be taken with each one of them. Furthermore, we have noticed that those systems have not the same behaviour for the four tests.

Table 4 presents a synthesis of the most signifiants results for CIS. We made the 
table using the results of the I.A.T., R.R.B.A.T.IV, R.R.B.A.T.VI, P.R.T. and S.D.A.T. protocoles. We have chosen to present the results as a mark between + + and - -, using $2 * 4$ differents threshold values for the rms error and max error $\left(\mathrm{T}_{0}, \mathrm{~T}_{0}{ }^{\prime}, \mathrm{T}_{1}, \mathrm{~T}_{1}{ }^{\prime}, \mathrm{T}_{2}, \mathrm{~T}_{2}{ }^{\prime}\right)$. For example, the threshold values for the R.R.B.A.T. IV are $(0.2,1,0.4,2,0.8,4)$ in $\mathrm{mm}$.

Table 4. Global results

\begin{tabular}{|r|c|c|c|c|c|}
\hline & Localizer & Optotrak & F P5000 & Polaris Active & Polaris Passive \\
\hline \hline Intrinsec Accuracy Test & y axis & + & 0 & 0 & + \\
\hline Relative Rigid Body Accuracy Test & IV & ++ & 0 & - & + \\
& VI & ++ & + & - & + \\
\hline Pivot Repeatability Test & & + & + & ++ & + \\
\hline Surface Digitization Accuracy Test & & ++ & 0 & - & + \\
\hline
\end{tabular}

\section{Conclusion}

This paper has presented a generic experimental protocol to evaluate localizer for CIS, and results of the evaluation for four optical localizers.

The experimental protocol is based on the required specifications for CIS applications and consists on four tests : I.A.T. which characterizes the intrinsec accuracy of the localizer, R.R.B.A.T. which characterizes the relative accuracy between two rigid bodies, P.R.T. which evaluates the repeatablity of the localizer to determine the tip of a pointer, and S.D.A.T. which characterizes the accuracy of surface digitization.

The second part of the paper, which is the evaluation using the defined protocol of four optical localizers (Optotrak, FP5000, Polaris active, Polaris passive) allows us to conclude on that the Optotrak is the most appropriated device for CIS if we consider only the global accuracy and robustness, but it is very expensive and its very high weight and size can cause many disagreements. FP5000 has robust measures. Accuracy is fair but sufficient. Nevertheless it is unusable for high-demanding applications. Active Polaris has a rather good accuracy but relative motion of two rigid bodies yield unacceptable errors. This is explained by the acquisition method. Passive Polaris has a good accuracy and is not affected at all by motions of the rigid bodies (due to its data capture principle). However, partial occlusions of markers provide small shifts and many rigid bodies configuration raise missing data.

Acknowledgement : Authors wish to thank Vincent Dessenne, Eric Bainville, Biao Chen and Nicolas Moreau for their contributions to integration and initial tests of localizers. This project is financially suppported by European Project IGOS II (Image Guided Orthopaedics Surgery). 


\section{References}

1. S. Lavallee, J. Troccaz, P. Sautot, B. Mazier, P. Cinquin, P. Merloz, and J.P. Chirossel. Computer assisted spine surgery using anatomy-based registration. In R. Taylor, S. Lavallee, G. Burdea, and R. Mosges, editors, Computer Integrated Surgery, chapter 32. MIT Press, Cambridge, MA, 1996.

2. F. Leitner, F. Picard, R. Minfelde, H.J. Schulz, P. Cinquin, and D. Saragaglia. Computer-assisted knee surgical total replacement. In J. Troccaz, E. Grimson, and R. Moesges, editors, CVRMed-MRCAS Proc., LNCS Series 1205, Grenoble, 1997. Springer-Verlag.

3. E. Bainville, P. Chaffanjon, and P. Cinquin. Computer generated visual assistance to a surgical intervention: the retroperitoneoscopy. In AAAI 94 Applications of Computer Vision in Medical Image Processing, Spring Symposium, Stanford, USA, 1994.

4. A. Mulder. Human movement tracking technology. http://fas.sfu.ca/cs/people/ResearchStaff/amulder/personal/vmi/HMTT.pub.html, 1994.

5. J. Atha. Current techniques for measuring motion. Ltf Applied Ergonimics, pages 245-257, 1984.

6. R. Rohling, P. Munger, J.M. Hollerbach, and T. Peters. Comparison of relative accuracy between a mechanical and an optical tracker for image-guided neurosurgery. Journal of image guided surgery, 1(1):30-34, 1995.

7. R. Marmulla, M. Hilbert, and H. Niederdellmann. Immanent precision of mechanical, infrared and laser guided navigation systems for CAS. In K. Inamura H.U. Lemke, M.W. Vannier, editor, Proceedings of the 11th International Symposium and Exhibition, pages 863-865. CAR'97, 1997.

8. A.R. Gunkel, W. Freysinger, W.F. Thumfart, and M.J. Truppe. Application of the ARTMA image-guided navigation system to endonasal sinus surgery. In Computer Assisted Radiology (CAR'95), pages 1147-1151, Berlin, 1995. Springer.

9. H.W. When and P.R. Beranger. Ultrasound-Based Robot Position Estimation. IEEE Transactions on Robotics and Automation, 13(5):682-692, 1997.

10. K.R. Smith, K.J. Frank, and R.D. Bucholz. The Neurostation: a highly accurate minimally invasive solution to frameless stereotactic neurosurgery. Computerized Medical Imaging and Graphics, 18(4):247-256, 1994.

11. A. Colchester, J. Zhao, K. Holton-Tainter, C. Henri, N. Maitland, P. Roberts, C. Harris, and R. Evans. Development and preliminary evaluation of VISLAN, a surgical planning and guidance system using intra-operative video imaging. Medical Image Analysis, 1(1):73-90, March 1996.

12. K.S. Arun, T.S. Huang, and S.D. Blostein. Least-squares fitting of two 3-D point sets. IEEE Trans. Pattern Anal. Machine Intell., PAMI-9(5):698-700, 1987.

13. S. Lavallee, P. Cinquin, and J. Troccaz. Computer Integrated Surgery and Therapy: State of the Art. In C. Roux and J.L. Coatrieux, editors, Contemporary Perspectives in Three-Dimensional Biomedical Imaging, chapter 10, pages 239-310. IOS Press, Amsterdam, NL, 1997. 